\title{
Renormalization approach for quantum-dot structures under strong alternating fields
}

\author{
P. A. Schulz \\ Instituto de Física 'Gleb Wataghin', Universidade Estadual de Campinas, 13083-970, Campinas, São Paulo, Brazil \\ P. H. Rivera and Nelson Studart \\ Departamento de Física, Universidade Federal de São Carlos, 13565-905, São Carlos, São Paulo, Brazil
}

\begin{abstract}
We develop a renormalization method for calculating the electronic structure of single and double quantum dots under intense ac fields. The nanostructures are emulated by lattice models with a clear continuum limit of the effective-mass and single-particle approximations. The coupling to the ac field is treated non-perturbatively by means of the Floquet Hamiltonian. The renormalization approach allows the study of dressed states of the nanoscopic system with realistic geometries as well arbitrary strong ac fields. We give examples of a single quantum dot, emphasizing the analysis of the effective-mass limit for lattice models, and double-dot structures, where we discuss the limit of the well used two-level approximation.
\end{abstract}

\section{INTRODUCTION}

The renormalization method, as a tool for studying the electronic structure of solids, has attracted attention already twenty years ago with the application to disordered one-dimensional systems. 1 . It is a powerful approach for systems whose complexity hinders the direct diagonalization of the corresponding Hamiltonian. At that time its complete usefulness could be hardly realized due to the difficulty in applying the method simultaneously in more than one spatial direction. More recently its suitability has been revealed for studying strongly anisotropic solids that started to deserve growing interest, like semiconductor superlattices and conducting polymers.

In the present work the renormalization method is applied to a different problem of increasing interest with the same formal structure: the dressed electronic spectra of quantum dot systems under intense ac fields.

In semiconductor technology, the continuous improvement on growth techniques opened possibilities to design systems that are in the quantum limit in all spatial directions, establishing an important and pioneering branch of what is nowadays called nanoscience. One of these devices, a double-quantum dot is a candidate for the elementary unit of the foreseen quantum computing. Two coupled quantum dots afterwards called as artificial molecules has heen the object of intensive research both theoretical 5 .

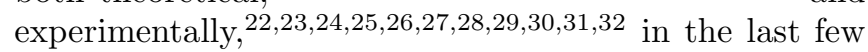
years.

Mesoscopic systems, like quantum dots and quantum dot arrays, are described by lattice models, treated in a tight-binding framework, emulating the continuum limit of the effective-mass approximation. 33 Lattice models have been used mainly in the context of disorder effects on electronic and transport properties of two-dimensional systems and quantum billiards. 34 On the other hand, there are reports in the literature on using lattice models in another situation, where they constitute also an appropriate approach, namely the simulation of arrays of quantum dots and antidots in the presence of a magnetic field 35 Realistic description of quantum dot nanostructures in the independent particle approximation interacting with intense ac fields becomes possible within the proposed renormalization-decimation procedure.

The coupling to an ac field is non-perturbatively included using the Floquet method, by means of a procedure introduced by Shirley. 3 The Floquet method has been widely used for the non-perturbative study of the interaction of atomic, molecular and semiconductor nanostructures systems with a strong ac electric field. The time-independent infinite matrix Hamiltonian obtained after the application of the Floquet-Fourier transformation over the time-dependent Schrödinger equation, describes entirely these processes without any further $a d$ hoc assumptions.

Therefore, the effect of an intense ac field on the electronic spectra of a nanostructure, like a quantum dot, is well described by an infinite Floquet matrix. However, due to the large vector basis used to define the system in a lattice-model tight-binding approximation, the necessary convergence criteria make the eigenvalue calculations practically impossible beyond the perturbative field intensity range. Moreover, we observe that the infinite matrix Hamiltonian structure resembles the structure of a linear chain matrix, where the "energy sites" are energy sub-matrices which corresponds to the Hamiltonian of the system plus or minus an associated multiple of the photon energy and the "hopping parameter" is the ac field coupling the sub-matrices. These relationships allow us to develop an interesting and promising renormalization approach whereby the actual dimension of the system to be calculated is reduced to the one of the lattice model for the bare system. Besides that, since the quantity calculated is the density of states, one gets a step further than by the direct diagonalization of the Floquet Hamiltonian, which provides only the quasi-energy spectra without the spectral modulation as a function of the field strength. The hierarchy of these quasi-energy spectra related to different photon replicas will also be discussed.

The paper is presented as follows. A brief introduction 
to the Floquet Hamiltonian is given in Sec. II, followed by a comprehensive description of the renormalization method applied to the system. In Sec. III, numerical calculations for single and double quantum dots are shown and discussed. We first focus on the validity of a lattice model for a quantum dot in the presence of intense ac fields. The second part is centered on the ac field dependence of the bonding and antibonding states of an artificial molecule, i.e., a double-quantum dot structure. In Sec. IV we present our conclusions.

\section{THEORY}

\section{A. The Floquet Hamiltonian}

The lower part of the energy spectrum of a mesoscopic system, like a quantum dot or quantum-dot array described in the framework of the effective-mass approximation, will be evaluated here using a tight-binding model for a square lattice of $s$-like orbitals, considering only nearest-neighbors interaction and a hopping parameter defined by $V=-\hbar^{2} /\left(2 m^{*} a^{2}\right)$, where $m^{*}$ is the effective mass and $a$ is the host lattice parameter.

The applied ac fields are parallel to one of the square sides. Hence, our model is described by the Hamiltonian $H=H_{0}+H_{\text {int }}$ where

$$
\begin{gathered}
H_{o}=\sum_{l_{1}, l_{2}} \epsilon_{l_{1}, l_{2}} \sigma_{l_{1}, l_{2}} \sigma_{l_{1}, l_{2}}^{\dagger}+\frac{V}{2} \sum_{l_{1}, l_{2}}\left[\sigma_{l_{1}, l_{2}} \sigma_{l_{1}+1, l_{2}}^{\dagger}+\right. \\
\left.\sigma_{l_{1}+1, l_{2}} \sigma_{l_{1}, l_{2}}^{\dagger}+\sigma_{l_{1}, l_{2}} \sigma_{l_{1}, l_{2}+1}^{\dagger}+\sigma_{l_{1}, l_{2}+1} \sigma_{l_{1}, l_{2}}^{\dagger}\right]
\end{gathered}
$$

and

$$
H_{i n t}=e a F \cos \omega t \sum_{l_{1}, l_{2}} \sigma_{l_{1}, l_{2}} l_{1} \sigma_{l_{1}, l_{2}}^{\dagger}
$$

with $\sigma_{l_{1}, l_{2}}=\mid l_{1}, l_{2}>$ and $\sigma_{l_{1}, l_{2}}^{\dagger}=<l_{1}, l_{2} \mid$. The atomic energies will be taken constant, $\epsilon_{l_{1}, l_{2}}=4|V|$, for all sites. $F$ and $\omega$ are the ac field amplitude and frequency respectively, and $e$ is the electron charge. The treatment of the time-dependent problem is based on Floquet states $\mid l_{1}, l_{2}, m>$ where $l_{1}, l_{2}$ are the site indexes and $m$ is the photon index. We follow the procedure developed by Shirley 36 which consists in a Fourier-Floquet transformation of the time-dependent Hamiltonian into a timeindependent infinite matrix which must be truncated. The matrix elements are

$$
\begin{gathered}
{\left[\left(\mathcal{E}-m \hbar \omega-\epsilon_{l_{1}, l_{2}}\right) \delta_{l_{1}^{\prime} l_{1}} \delta_{l_{2}^{\prime} l_{2}}-\frac{V}{2}\left\{\left(\delta_{l_{1}^{\prime}, l_{1}-1}+\delta_{l_{1}^{\prime}, l_{1}+1}\right) \delta_{l_{2}^{\prime} l_{2}}\right.\right.} \\
\left.\left.+\left(\delta_{l_{2}^{\prime}, l_{2}-1}+\delta_{l_{2}^{\prime}, l_{2}+1}\right) \delta_{l_{1}^{\prime} l_{1}}\right\}\right] \delta_{m^{\prime} m} \\
=F_{1} l_{1} \delta_{l_{1}^{\prime} l_{1}} \delta_{l_{2}^{\prime} l_{2}}\left(\delta_{m^{\prime}, m-1}+\delta_{m^{\prime}, m+1}\right)
\end{gathered}
$$

where $F_{1}=\frac{1}{2} e a F$. The dimension of the matrix is $L_{1} \times L_{2}(2 M+1)$, where $L_{1}$ and $L_{2}$ are the maximum number of atomic sites, while $M$ is the maximum photon index. We choose $M$ in order to satisfy a convergence condition: symmetric spectra relative to the edges of the quasi Brillouin zones (QBZs). The first QBZ is spanned in the range $-\hbar \omega / 2 \leq \mathcal{E} \leq \hbar \omega / 2$.

The truncated Floquet matrix resembles a linear chain matrix in the form

$$
\left(\begin{array}{ccccccccc}
\mathrm{E}^{M} & \mathcal{F} & & & & & & & \\
\mathcal{F} & \mathrm{E}^{M-1} & \mathcal{F} & & & & & & \\
& & \ddots & & & & & & \\
& & \mathcal{F} & \mathrm{E}^{1} & \mathcal{F} & & & & \\
& & & \mathcal{F} & \mathrm{E}^{0} & \mathcal{F} & & & \\
& & & & \mathcal{F} & \mathrm{E}^{-1} & \mathcal{F} & & \\
& & & & & \ddots & & & \\
& & & & & & \mathcal{F} & \mathrm{E}^{-M+1} & \mathcal{F} \\
& & & & & & & \mathcal{F} & \mathrm{E}^{-M}
\end{array}\right)
$$

where $\mathrm{E}^{m}=\mathcal{E}-(\mathrm{S}+m \hbar \omega)$ is a $L_{1} \times L_{2}$ matrix, with $\mathcal{E}$ being the quasi-energy spectrum and $\mathrm{S}$, is the $L_{1} \times L_{2}$ system matrix. For other hand, the $L_{1} \times L_{2}$ diagonal matrix $\mathcal{F}$ represents the coupling of the system with the ac electric field and is given by

$$
\mathcal{F}=F_{1} l_{1} \delta_{l_{1}^{\prime} l_{1}} \delta_{l_{2}^{\prime} l_{2}}
$$

A time-independent Schrödinger equation defined in terms of Green functions as 37

$$
(\mathcal{E}-H) G=1
$$

can be associated to the Floquet matrix, as we will show next.

\section{B. Renormalization Method}

Equation (6) can be projected to the photon Hilbert spaces $<n \mid$ and $\mid m>$, resulting in

$$
\sum_{k}<n|(\mathcal{E}-H)| k>G_{k m}=\delta_{n m}
$$

Expanding this equation, considering $\mathcal{E} \rightarrow \mathcal{E}+i \eta$, where $\eta \rightarrow 0$, eliminating the intermediate index sites, making some substitutions, and repeating the decimation process successively we arrive to the general expression, for any $\xi \geq 1$ order decimation of the Eq. (ఫ):

$$
E_{n}^{\xi} G_{n 0}=\delta_{n 0}+\mathcal{F}_{n-2^{\xi}, n}^{\xi} G_{n-2^{\xi}, 0}+\mathcal{F}_{n+2^{\xi}, n}^{\xi} G_{n+2^{\xi}, 0}
$$

where 


$$
\begin{gathered}
E_{n}^{\xi}=E_{n}^{\xi-1}-\mathcal{F}_{n-2^{\xi-1}, n}^{\xi-1} \frac{1}{E_{n-2^{\xi-1}}^{\xi-1}} \mathcal{F}_{n, n-2^{\xi-1}}^{\xi-1}- \\
\mathcal{F}_{n+2^{\xi-1}, n}^{\xi-1} \frac{1}{E_{n+2^{\xi-1}}^{\xi-1}} \mathcal{F}_{n, n+2^{\xi-1}}^{\xi-1} \\
\mathcal{F}_{n-2^{\xi}, n}^{\xi}=\mathcal{F}_{n-2^{\xi-1}, n}^{\xi-1} \frac{1}{E_{n-2^{\xi-1}}^{\xi-1}} \mathcal{F}_{n-2^{\xi}, n-2^{\xi-1}}^{\xi-1} \\
\mathcal{F}_{n+2^{\xi, n}}^{\xi}=\mathcal{F}_{n+2^{\xi-1}, n}^{\xi-1} \frac{1}{E_{n+2^{\xi-1}}^{\xi-1}} \mathcal{F}_{n+2^{\xi}, n+2^{\xi-1}}^{\xi-1} .
\end{gathered}
$$

The Green function $G_{00}$ after the first decimation is given by

$$
\begin{aligned}
G_{00}= & {\left[\left(E_{0}-\mathcal{F} \frac{1}{E_{-1}} \mathcal{F}-\mathcal{F} \frac{1}{E_{1}} \mathcal{F}\right)-\right.} \\
& \left(\mathcal{F} \frac{1}{E_{-1}} \mathcal{F}\right) \frac{1}{A_{-2}}\left(\mathcal{F} \frac{1}{E_{-1}} \mathcal{F}\right)- \\
& \left.\left(\mathcal{F} \frac{1}{E_{1}} \mathcal{F}\right) \frac{1}{A_{2}}\left(\mathcal{F} \frac{1}{E_{1}} \mathcal{F}\right)\right]^{-1}
\end{aligned}
$$

where

$$
\begin{aligned}
A_{-2}= & \left(E_{-2}-\mathcal{F} \frac{1}{E_{-1}} \mathcal{F}-\mathcal{F} \frac{1}{E_{-3}} \mathcal{F}\right)- \\
& \left(\mathcal{F} \frac{1}{E_{-3}} \mathcal{F}\right) \frac{1}{B_{-4}}\left(\mathcal{F} \frac{1}{E_{-3}} \mathcal{F}\right) ; \\
A_{2}= & \left(E_{2}-\mathcal{F} \frac{1}{E_{1}} \mathcal{F}-\mathcal{F} \frac{1}{E_{3}} \mathcal{F}\right)- \\
& \left(\mathcal{F} \frac{1}{E_{3}} \mathcal{F}\right) \frac{1}{B_{4}}\left(\mathcal{F} \frac{1}{E_{3}} \mathcal{F}\right)
\end{aligned}
$$

The density of states of Floquet is expressed as

$$
\rho(\mathcal{E}+i \eta)=\lim _{\eta \rightarrow 0}\left(-\frac{1}{\pi} \operatorname{Im}\left[\operatorname{Tr} G_{00}\right]\right) .
$$

\section{ARTIFICIAL ATOMS AND MOLECULES}

\section{A. Quantum dot under intense ac field}

As a starting point we calculate the quasi-density of states of quantum dots as a function of the ac field intensity. We choose a square and a rounded geometry to represent the quantum dot as shown in Fig. 1. The square quantum dot consists of an array of $6 \times 6$ atomic sites, Fig. 11(a). A more realistic geometry is given in Fig.1(b), which will be used for the study of double-dot systems in this work. It should be noticed that the area of this geometry (37 sites) is comparable with the square one if we use the same tight-binding parameters given below. Such lattice models exhibit a particle-hole symmetry in the electronic structure and are usually thought as simple, although useful, approximations for superlattices or arrays of quantum dots, where each quantum well or quantum dot is represented by a lattice site, respectively. This extreme lattice limit has been used for studying qualitatively the effect of intense ac fields on superlattice minibands.38 On the other hand, lattice models may be useful in calculating the lower part of electronic systems well described by the effective mass approximation. In the present work, the tight-binding hopping parameter is chosen in order to emulate the electron effective mass for the GaAs bottom of the conduction band, $m^{*}=0.067 m_{0}$. Since $V=-\hbar^{2} /\left(2 m^{*} a^{2}\right), V=-0.142 \mathrm{eV}$ for a model lattice parameter of $a=20 \AA$. This leads to a quantum dot with a lateral width of $L=120 \AA$, an order of magnitude lower than typical dimensions of actual quantum dots fabricated by lithographic methods. We intend to illustrate the method with the present calculations. Nevertheless, all conclusions can be traced back to larger systems by an appropriate scaling of the energy parameters.

The first point to be addressed here is establishing the limit between the extreme-lattice and effective-mass limits. This is achieved by following the evolution of the quasi-density of states as a function of the ac field intensity with frequencies of the order of the electronic band width. In Fig 2 we show the contour plot of the density of states as a function of the field intensity for the bottom half of the first QBZ. We recall that, for the chosen hopping parameter, the band width for a square array of single atomic $s$-like showing particle-hole symmetry is given by $\Delta E=8|V|=1.136 \mathrm{eV}$. Here the field frequency is $\hbar \omega=1.0 \mathrm{eV}$. The square quantum dot, even though a textbook example, is very useful to understand the breakdown of the effective-mass limit. At zero field intensity the energy spectrum of a $2 \mathrm{D}$ square potential well with $L=120 \AA$ - a quantum dot - is clearly identified, as well as the breakdown of degeneracies due to the applied ac field. The ac Stark shifts lead to crossings at $e a F / \hbar \omega \approx 2.4$, corresponding to a dynamic localization along the chains perpendicular to the field direction. This is the limit where the host lattice effects are already predominant, i.e., the discrete basis of atomic orbitals does not emulate the effective-mass approximation anymore and the length scale is given by the host lattice parameter $a$ and not by the lateral width $L$ of the quantum dot. Indeed, the model simulates in this limit a square array of quantum dots, each one represented by a single site, resembling the spectra that would be expected for coupled chains, where each chain mimics the dynamic localization in superlattice minibands.

This result for a square array is a good guide for characterizing the density of states as a function of the field intensity of a quantum dot with lower symmetry, Fig.1(b), as can be seen in the equivalent plot shown in Fig.2(b), also for $\hbar \omega=1.0 \mathrm{eV}$. The dynamic localization at the ex- 
treme lattice limit is less defined due to the fact that the atomic site chains perpendicular to the field direction are not equivalent as in the square array. Furthermore some of the degeneracies are already broken in the absence of the ac field.

One clear advantage of the method can already be seen in Fig. 2. By diagonalizing the Floquet Hamiltonian, the quasi-energy spectra are depicted in the so called QBZs, each of them reproducing the spectrum with the energy shifted by integer multiples of the photon energy. The overlap of these photon replicas makes the interpretation of the spectrum rather cumbersome, specially for strong overlaps, which are unavoidable for low frequencies. The present renormalization approach indicates that the quasi-energy spectrum, modulated by the field dependent density of states for higher or lower photon replicas becomes relevant only with increasing field intensity. This effect is verified at the bottom of Figs. 2(a) and 2(b). The spectrum replica lowered by one photon energy shows a negligible contribution at low field. On the other hand, in both figures we see no significant modulation of the density of states in the low field limit within the entire band of the depicted zero photon replica, due to the high frequency considered.

The main interest, however, is the effective-mass limit, i.e., the energy bottom of each photon replica at low fields in the scale of Fig. 2, as well as low frequencies, that would couple only these few low-energy states. The scaling of these quantities shows the suitability of the present method, as will be seen in the following discussion on double quantum dots.

\section{B. Double Quantum dot}

Our double-quantum dot system is based on the quantum dot shown in Fig. 1 (b). The coupling between dots is of free choice and we consider a simple connection of both dots by the same hopping parameters considered so far, as illustrated in Fig. 3. Such configuration is an example of strong inter-dot coupling. The corresponding quasi-density of states plot as a function of the field intensity for the same high field frequency, $\hbar \omega=1.0 \mathrm{eV}$, as in Fig. 2 is shown in Fig. 14(a). The quasi-density of states spectrum is very similar with the appearance of the expected energy splitting due to the coupling among quantum dot states. The structure of the energy splitting may be rather complex, considering the coupling of initially degenerate states in each quantum dot. A covalent-like binding, with a splitting between a bonding and antibonding states, is well defined for the inter-dot coupling between the lowest state in each quantum dot. Therefore, from now on we will focus exclusively on the energy bottom of the spectra, the continuum low-field intensity limit, in order to analyze the lowest pair of split double-quantum dot states.

In Fig. A(b) we have the quasi-density of states for the lowest pair of molecular states. Both bonding and an- tibonding states shift rigidly upwards in energy. Here a modulation of the density of states is seen. By increasing the field intensity, the density of states diminishes, with increasing contribution of higher and lower photon replica (not shown). Since the frequency of the field is very high $\hbar \omega=1 \mathrm{eV}$, all states of the system are mixed by the field and no typical two-level behavior is observed. The rigid energy shift may be seen already as a lattice effect, since for high field intensities, the dynamic localization for the host lattice is observed, Fig. 4. (a).

A clear covalent picture is revealed for much lower frequencies, in the range of $\hbar \omega \approx 10 \mathrm{meV}$, which is of the order of the tunnel splitting between the lowest pair of states in the bare "molecule" for the chosen parameters: $\Delta_{\text {split }}=7.1 \mathrm{meV}$. For this frequency range the coupling to higher molecular states is negligible, since the third molecular state is about $50 \mathrm{meV}$ above the antibonding state. Lattice effects are also absent since the dynamic localization effects on the host lattice are relevant for field frequencies of the order of the entire spectral width, which is two orders of magnitude larger than the energy scale of interest given by the tunnel splitting. The present calculations are exact, since we are in an independent particle approximation, for an artificial $\mathrm{H}_{2}^{+}$ molecule. Within this parameter range, the lowest pair of states of the double-quantum dot behave as a two-level system, as will be discussed next. We are going to switch the field frequency from above to below the tunnel splitting energy. The quasi-density of states plots will also be compared to the corresponding quasi-energy spectra of the equivalent two level system, obtained by diagonalizing the Floquet Hamiltonian.

In Fig. 5 the evolution of the artificial $\mathrm{H}_{2}^{+}$bonding and antibonding states as a function of the field intensity is shown for a frequency $\hbar \omega=10 \mathrm{meV}>\Delta_{\text {split }}$. In Fig. 5(a) we see the quasi-density of states around the main "photon replica", which shows the highest intensity for low fields. The next important branches of the quasidensity of states are the bonding state plus one photon and the antibonding minus one photon. With increasing the intensity, the splitting between the zero-photon bonding and antibonding states diminishes down to a crossing at $e a F / \hbar \omega \approx 0.3$. This resembles the dynamic localization regime for superlattice minibands. 38 The branches of the quasi-density of states in Fig. 5(a) can be mapped on the quasi-energy spectrum of a two-level system, emulated by two atomic sites with fitted tight-binding parameters. This spectrum is a function of a field intensity defined by $e d F / \hbar \omega$, where $d$ is the distance between the two effective atomic sites. The correspondence between Figs. 5(a) and 5(b) is satisfied by properly scaling $d \approx 7 a$. Having in mind the double quantum dot of Fig. $3, d_{0}=7 a$ is the distance between the centers of the dots, i.e. "the bond length of the molecule".

The equivalent situation, for a frequency given by $\hbar \omega=7 \mathrm{meV}$, therefore near the resonance situation $\Delta_{\text {split }} \approx \hbar \omega$, is shown in Fig.6. Here we clearly see that the bonding and antibonding states evolve in Rabi 
sidebands 39 This behavior can also be mapped on an effective two-level system, Fig. 6(b). The effective distance between the two effective atomic sites is close to the "molecular bond length" $d_{0}=7 a$, like for frequencies higher than the tunnel splitting, Fig.5.

The quasi-resonant case may be analyzed directly from the Rabi frequency at the crossing of the sidebands in Fig. 6(a). At the crossing $\hbar \omega_{R}=\Delta_{\text {split }}$, where $\omega_{R}=$ $d_{R} F / \hbar$ with $d_{R}$ being the dipole matrix element. Since $\Delta_{\text {split }}=\hbar \omega$, one has that $e a F \approx 0.3 \hbar \omega_{R}$, resulting in a dipole matrix element $d_{R} \approx 3.33 a$ and $2 d_{R}=6.6 a$, in good agreement with the "molecular length" $d_{0}=7 a$.

In Fig. 7 we show the situation for a field frequency $\hbar \omega=5 \mathrm{meV}$ lower than the bare tunnelling splitting. The zero-photon molecular states show an ac Stark shift, Fig. 7(a). The dressed tunnel splitting increases with field intensity up to the first important anticrossing at $e a F / \hbar \omega=1$. Now the mapping on an effective two-level system, Fig.7(b), occurs for an effective distance $d \approx$ 3.5 , nearly half the nominal molecular bond length. This shrinking of the effective distance is, however, compatible with the increasing of the tunnel splitting.

\section{CONCLUSIONS}

The present results demonstrate the usefulness of the renormalization-decimation method applied to the Flo- quet Hamiltonian. Such a procedure makes possible the calculation of quasi-density of states of realistic geometries for nanostructures under intense ac fields described by lattice models. The matrix dimension in the renormalization method is $L_{1} \times L_{2}$, while the direct diagonalization has to handle with matrix dimension of $(2 M+1) \times\left(L_{1} \times L_{2}\right)$. This is of paramount importance if we must consider $M \approx 100$ for $L_{1,2}>20$ in order to simulate realistic mesoscopic systems 38 The examples shown here for double quantum dots, although heuristic, point out interesting dependence of the dressed electronic structure on the field frequency. A natural extension of the work is the analysis of the local quasi-density of states, as well as the study of asymmetric doublequantum dots, a situation for which the density of states reveals actual tunnelling properties, in order to discuss transport measurements in such systems.

\section{Acknowledgments}

It is a pleasure to thank H. S. Brandi for suggesting the possibility of decimating the Floquet Hamiltonian, as well as many encouraging discussions at the beginning of this work. The work was supported by Fundação de Amparo à Pesquisa do Estado de São Paulo (FAPESP) and Conselho Nacional de Desenvolvimento Científico e Tecnológico (CNPq).
1 H. Aoki, J. Phys. C: Solid State Phys. 13, 3369 (1980).

2 C. E. T. G. DaSilva and B. Koiller, Solid State Comm. 40, 215 (1981).

3 R. Farchioni, P. Vignolo, and G. Grosso, Phys. Rev. B 60, 15705 (1999).

${ }^{4}$ L.Kouwenhoven, Science 268, 1440 (1995).

${ }^{5}$ G. W. Bryant, Phys. Rev. B 44, 3064 (1991).

${ }^{6}$ G. Klimeck, G. Chen, and S. Data, Phys. Rev. B 50, 2316 (1994).

7 C. Niu, L. Liu, and T. Lin, Phys. Rev. B 51, 5130 (1995).

8 J. J. Palacios and P. Hawrylak, Phys. Rev. B 51, 1769 (1995).

9 J. H. Oh, K. J. Chang, G. Ihm, and S. J. Lee, Phys. Rev. B 53, R13264 (1996).

10 R. Kotlyar and S. D. Sarma, Phys. Rev. B 55, R10205 (1997).

11 T. Aono and K. Kawamura, Jpn. J. Appl. Phys. 36, 3936 (1997).

12 H. Aoki, Physica E 1, 198 (1997).

13 T. Pohjola, J. K. M. M. Salomaa, J. Schmid, H. Schoeller, and G. Schön, Europhys. Lett. 40, 189 (1997).

14 P. Brune, C. Bruder, and H. Schoeller, Phys. Rev. B 56, 4730 (1997).

15 O. Mayrock, S. A. Mikhailov, T. Darnhofer, and U. Rössler, Phys. Rev. B 56, 15760 (1997).

16 N. E. Kaputkina and Y. E. Lozovik, Physics of the Solid State 40, 1929 (1998).

17 H. Imamura, H. Aoki, and P. A. Maksym, Phys. Rev. B 57, R4257 (1998).
18 Y. Asano, Phys. Rev. B 58, 1414 (1998).

19 W. Xie and C. Chen, Phys. Lett. A 245, 297 (1998).

20 C. A. Stafford, R. Kotlyar, and S. D. Sarma, Phys. Rev. B 58, 7091 (1998).

21 B. Partoens and F. M. Peeters, Physica B 298, 282 (2001).

22 A. Lorke and J. P. Kotthaus, Phys. Rev. Lett. 64, 2559 (1990).

23 M. Tewordt, R. J. F. Hughes, L. M. Moreno, J. T. Nichols, M. J. K. H. Asahi, V. J. Law, D. A. Ritchie, J. E. F. Frost, G. A. C. Jones, and M. Pepper, Phys. Rev. B 49, 8071 (1994).

24 F. R. Waugh, M. J. Berry, D. J. Mar, R. M. Westerveit, K. L. Campman, and A. C. Gossard, Phys. Rev. Lett. 75, 705 (1995).

25 D. Dixon, L. P. Kouwenhoven, P. L. McEuen, Y. Nagamune, J. Motohisa, and H. Sakaki, Phys. Rev. B 53, 12625 (1996).

26 R. H. Blick, R. J. Haug, J. Weiss, D. Pfannkuche, K. v. Klitzing, and K. Eberl, Phys. Rev. B 53, 7899 (1996).

27 T. Schmidt, R. J. Haug, K. v. Klitzing, A. Förster, and H. Lüth, Phys. Rev. Lett. 78, 1544 (1997).

${ }^{28}$ R. H. Blick, D. W. van der Weide, R. J. Haug, and K. Eberl, Phys. Rev. Lett. 81, 689 (1998).

29 R. H. Blick, D. Pfannkuche, R. J. Haug, K. v. Klitzing, and K. Eberl, Phys. Rev. Lett. 80, 4032 (1998).

30 T. H. Oosterkamp, T. Fujisawa, W. G. van der Wiel, K. Ishibashi, R. V. H. S. tarucha, and L. P. Kouwenhoven, Nature 395, 873 (1998).

31 T. H. Oosterkamp, S. F. Godijn, M. J. Uilenreef, Y. V. 
Nazarov, N. C. van der Vaart, and L. P. Kouwenhoven, Phys. Rev. Lett. 80, 4951 (1998).

32 T. Fujisawa, T. H. Oosterkamp, W. G. van der Wiel, B. W. Broer, R. Aguado, S. Tarucha, and L. P. Kouwenhoven, Science 282, 932 (1998).

33 B. Huckestein, Rev. Mod. Phys. 67, 357 (1995).

34 E. Louis and J. A. Verges, Phys. Rev. B 63, 115310 (2001).

35 I. V. Zozoulenko, F. A. Maa $\phi$, and E. H. Hauge, Phys. Rev. B 51, 7058 (1995).

36 J. H. Shirley, Phys. Rev. 138, B979 (1963).

37 E. N. Economou, Green's Functions in Quantum Physics (Springer-Verlag, Berlin, 1983).

38 P. H. Rivera and P. A. Schulz, Phys. Rev. B 61, R7865 (2000).

39 H. Haug and S. W. Koch, Quantum Theory of the Optical and Electronic Properties of Semiconductors (World Scientific, Singapur, 1990).
FIG. 1: Quantum dot geometries:(a) square and (b) rounded square dots, where $a$ is the host lattice parameter.

FIG. 2: Density of states for the (a) square and (b) rounded dots as a function of the field intensity. The field frequency is $\hbar \omega=1.0 \mathrm{eV}$. Only the bottom half of the first QZB is shown (see text).

FIG. 3: Double quantum-dot geometry based on rounded square dots, where $d_{0}$ is the "bond length". 
FIG. 4: Density of states of the double quantum-dot shown in Fig. 3 as a function of field intensity at high frequency $\hbar \omega=1$ $\mathrm{eV}$, (a) the bottom half of the first QBZ and (b) the bonding and antibonding states.

FIG. 5: (a) Density of states of a double quantum-dot as a function of the field intensity for a frequency, $\hbar \omega=10 \mathrm{meV}$; (b) spectrum of a equivalent two-level system, simulated with a dipole distance $d \approx d_{0}$, in the dynamic localization regime.

FIG. 6: (a) Same as Fig. 5 for $\hbar \omega=7 \mathrm{meV}$, and (b) same as Fig. 5 near the Rabi resonance.

FIG. 7: (a) same as Fig. Ffor $\hbar \omega=5 \mathrm{meV}$, and (b) same as Fig. 5 in the ac Stark regime. 


$$
\begin{array}{ccccc}
\bullet & \bullet & \bullet & \bullet \\
\bullet & \bullet & \bullet & \bullet & \bullet \\
\bullet & \bullet & \bullet & \bullet & \bullet \\
\bullet & \bullet & \bullet & \bullet & \bullet \\
\bullet \\
\bullet & \bullet & \bullet & \bullet & \bullet \\
\bullet & \bullet & \bullet & \bullet & \bullet \\
& (\mathrm{a})
\end{array}
$$




$$
\begin{array}{rrrrrrr} 
& 0 & 0 & 0 & & \\
0 & 0 & 0 & 0 & 0 & \\
0 & 0 & 0 & 0 & 0 & 0 & 0 \\
0 & 0 & 0 & 0 & 0 & 0 & 0 \\
0 & 0 & 0 & 0 & 0 & 0 & 0 \\
0 & 0 & 0 & 0 & 0 \\
& 0 & 0 & 0 & &
\end{array}
$$

(b) 
SOO 607
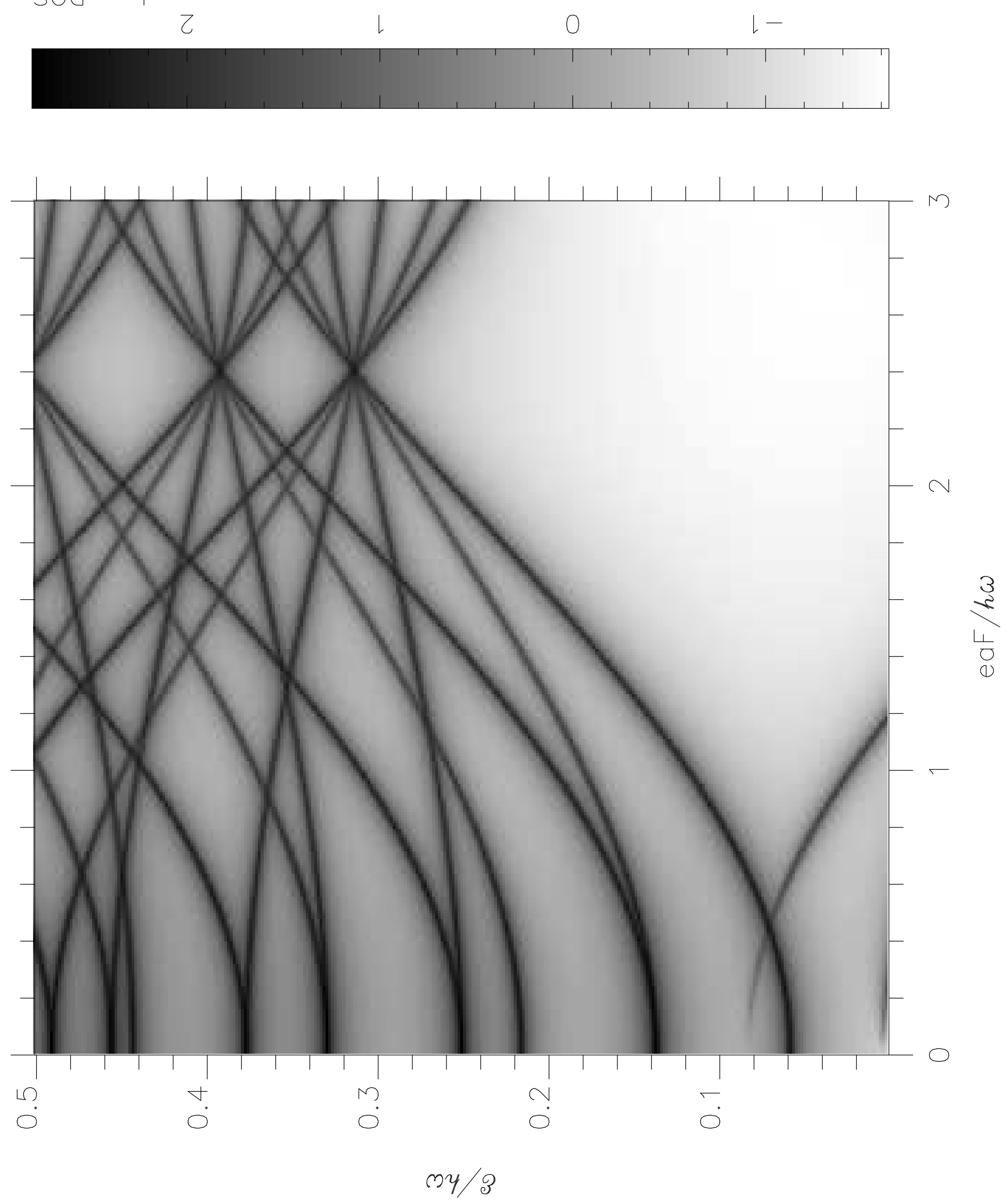
soo 507
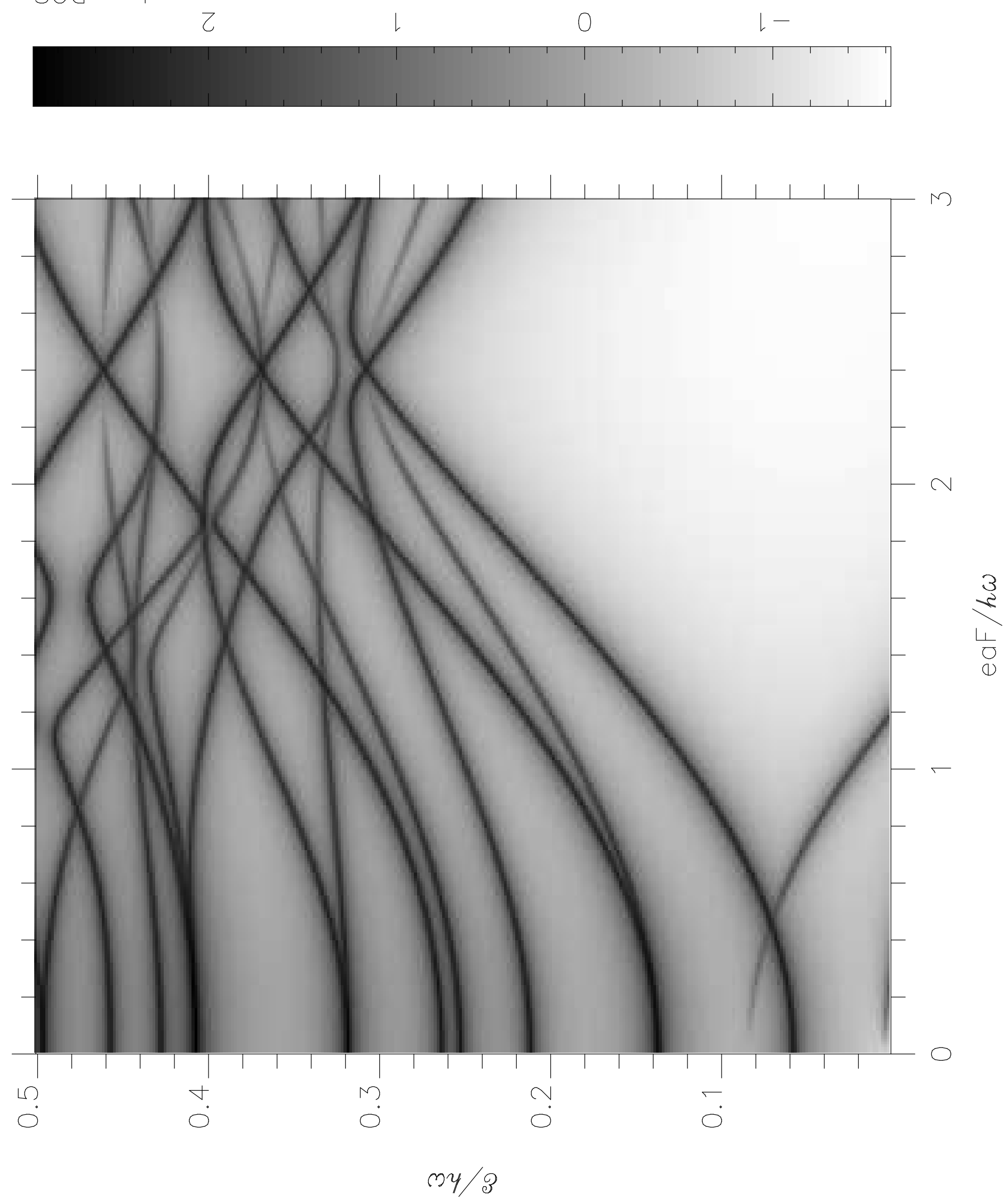


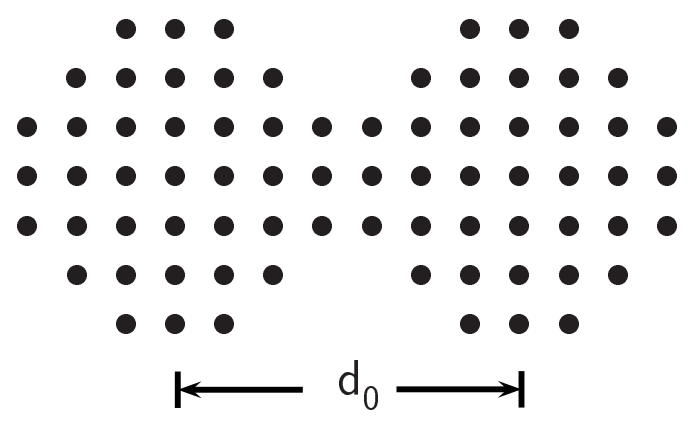


sod 607 乙

$0 \quad 1-$

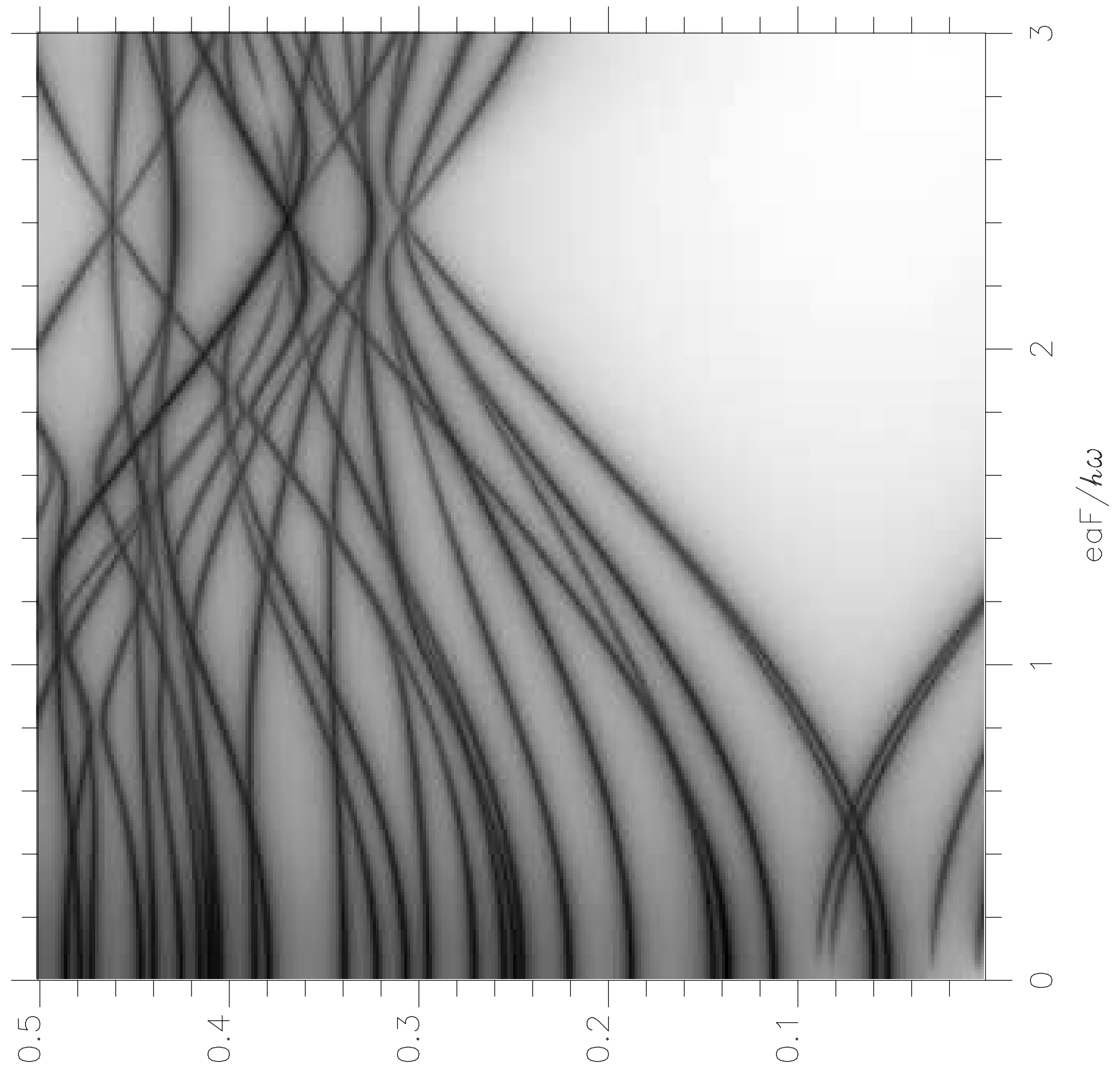


soo
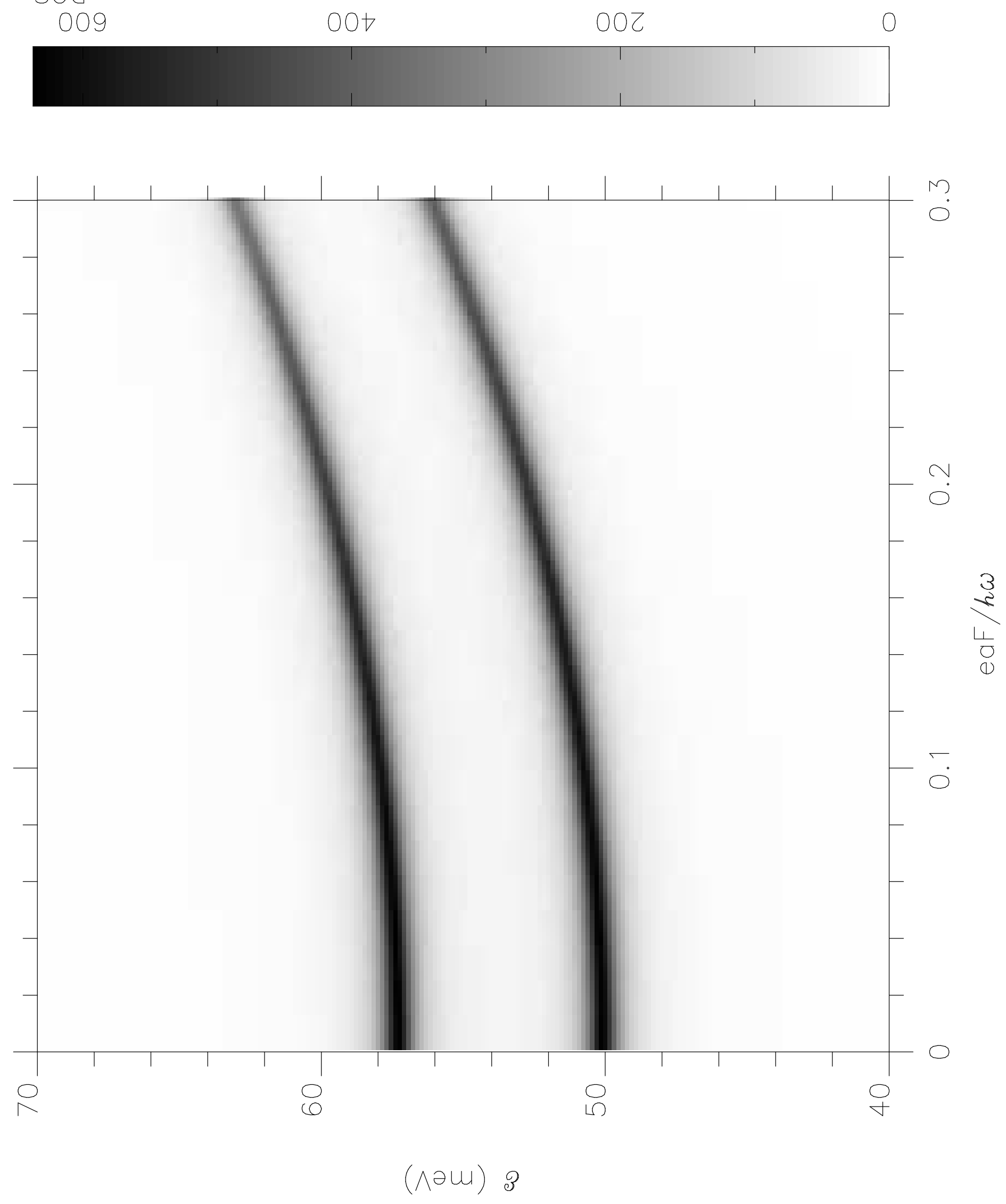

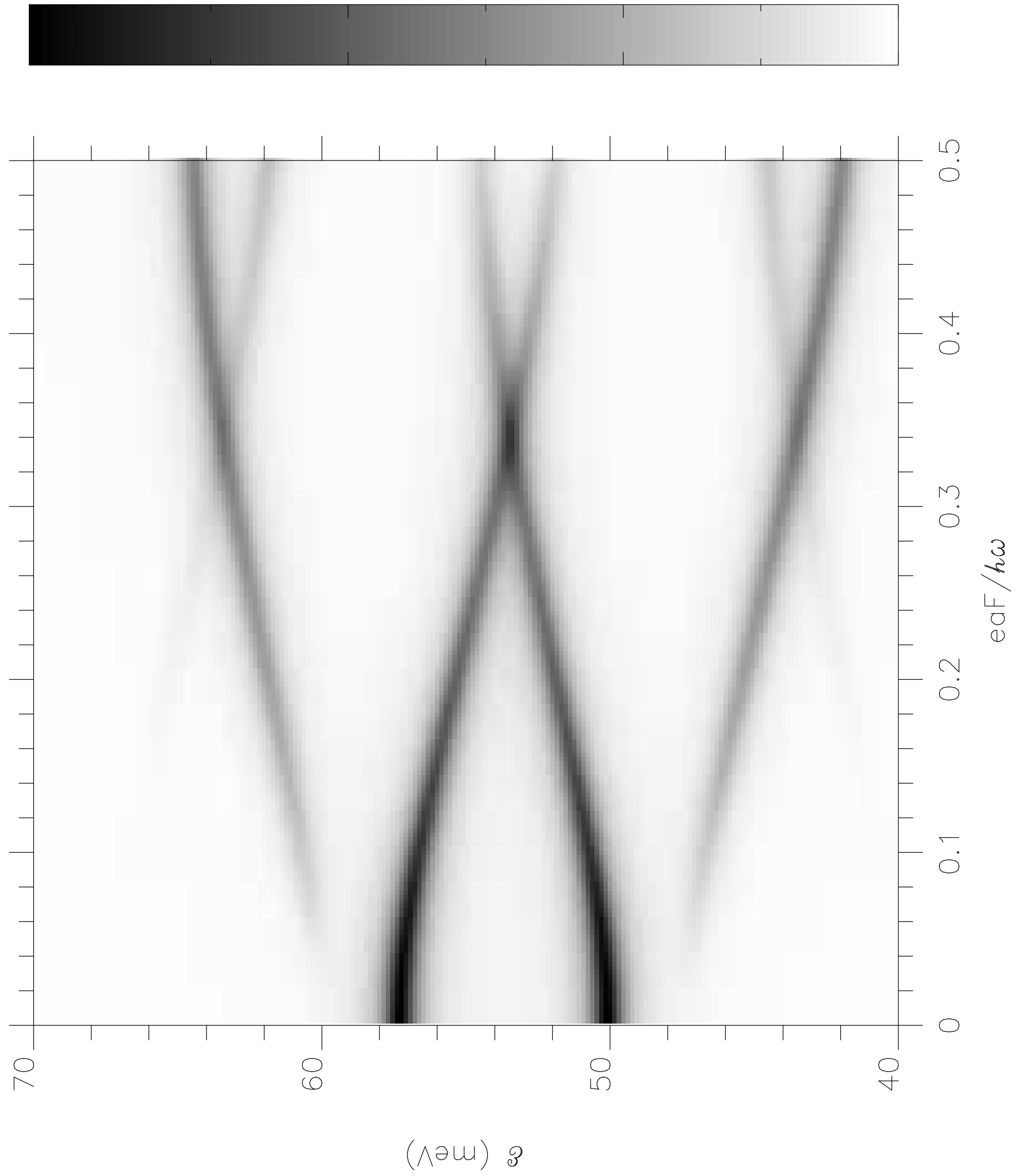


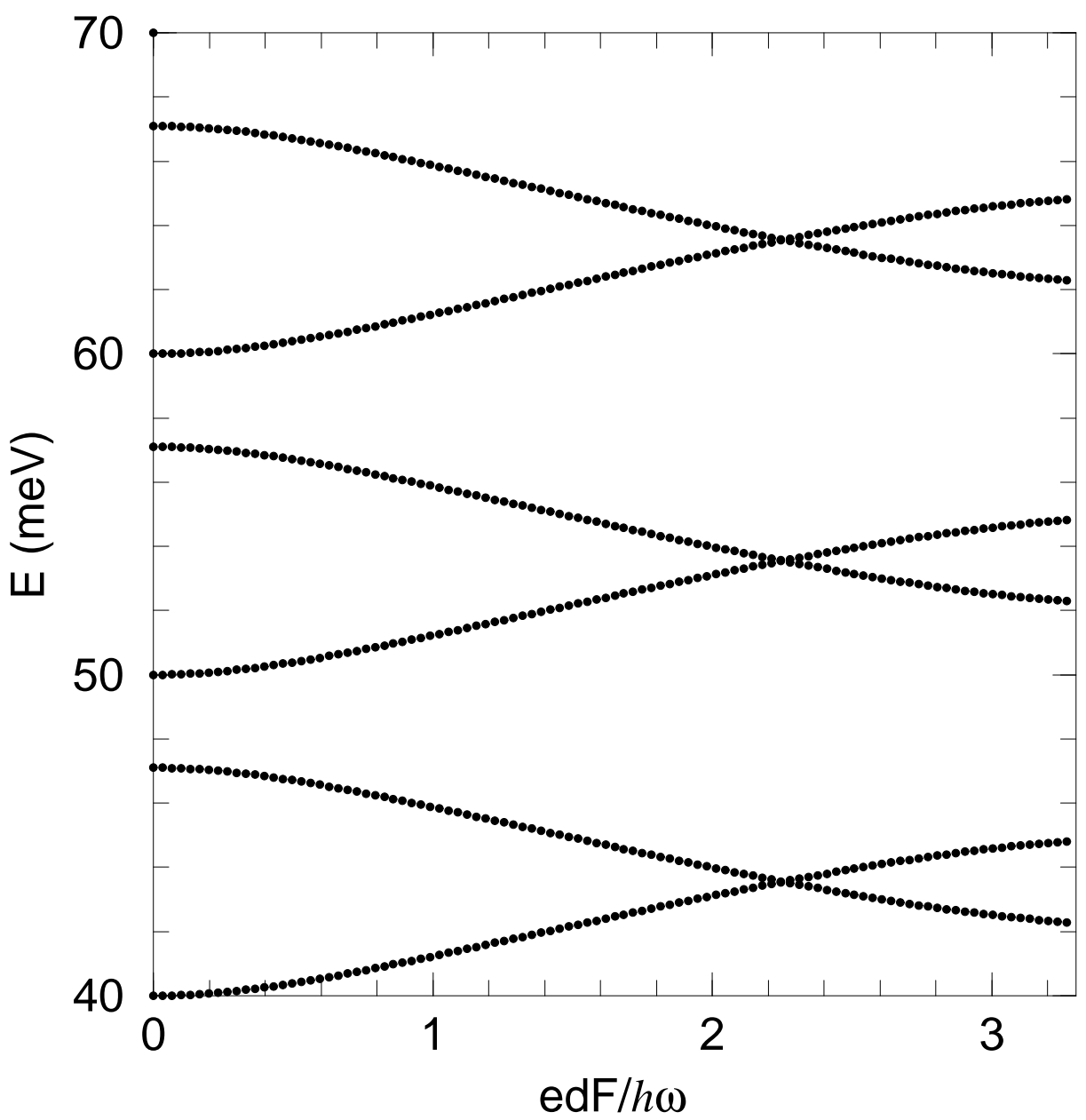



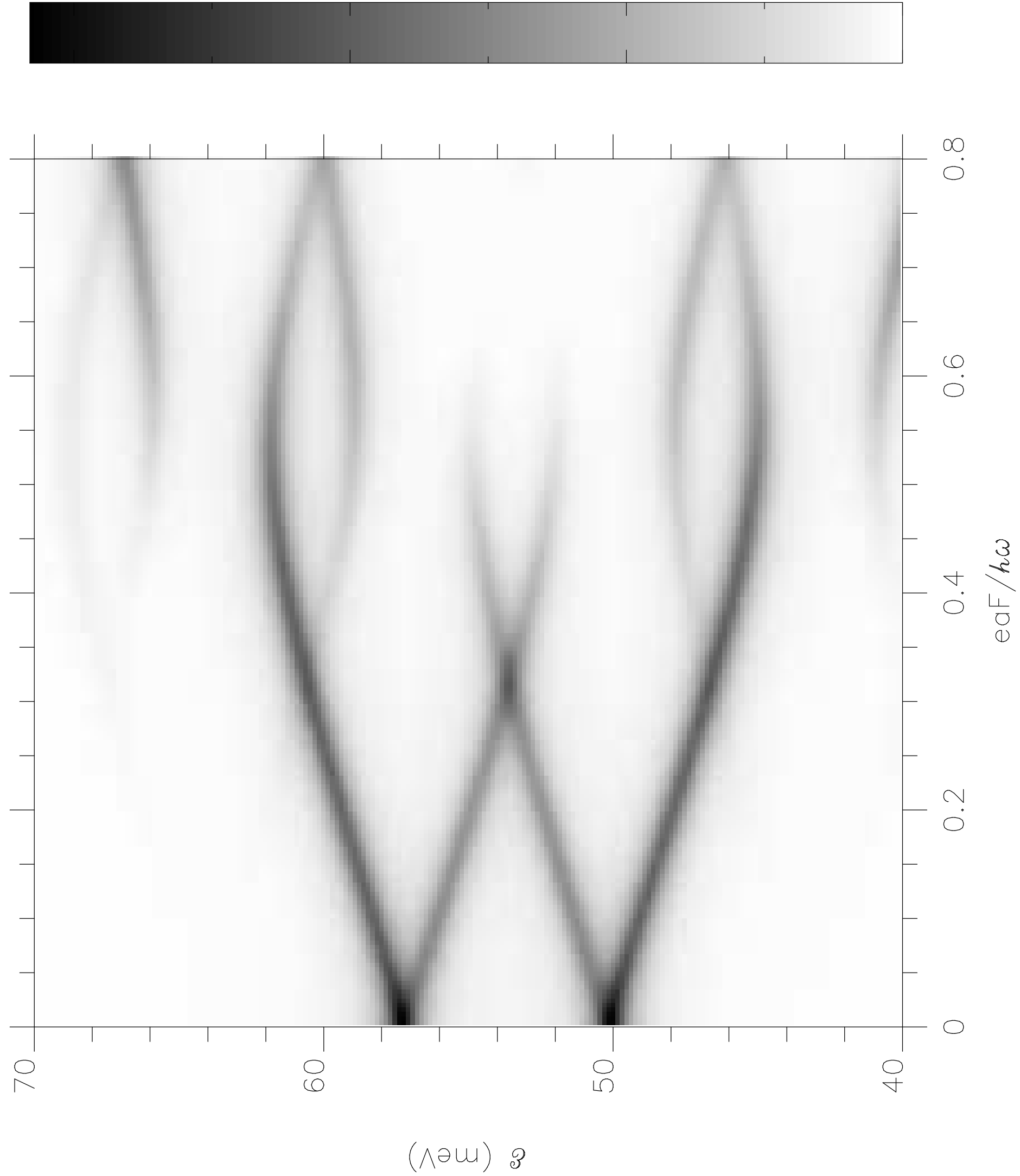


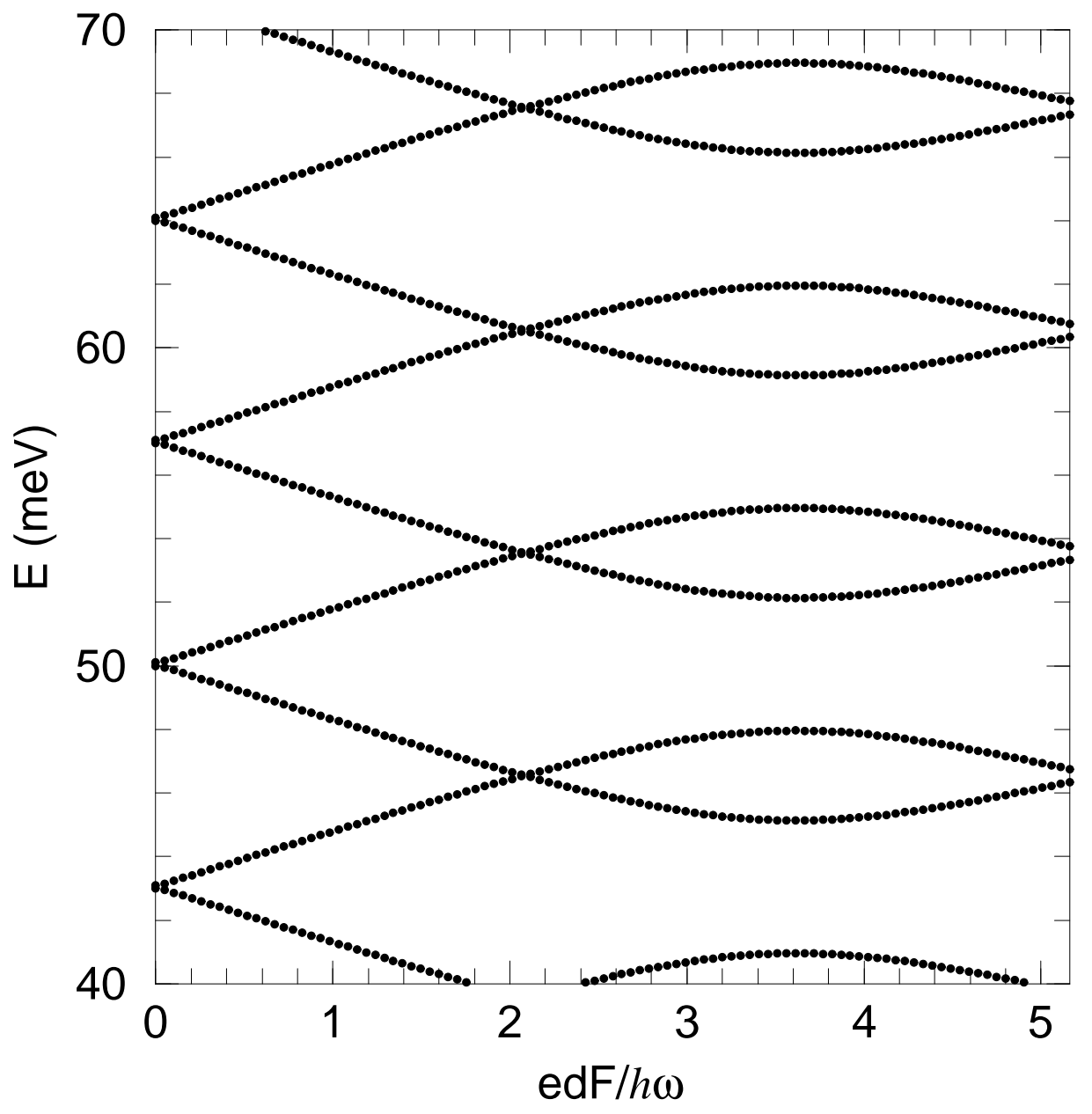



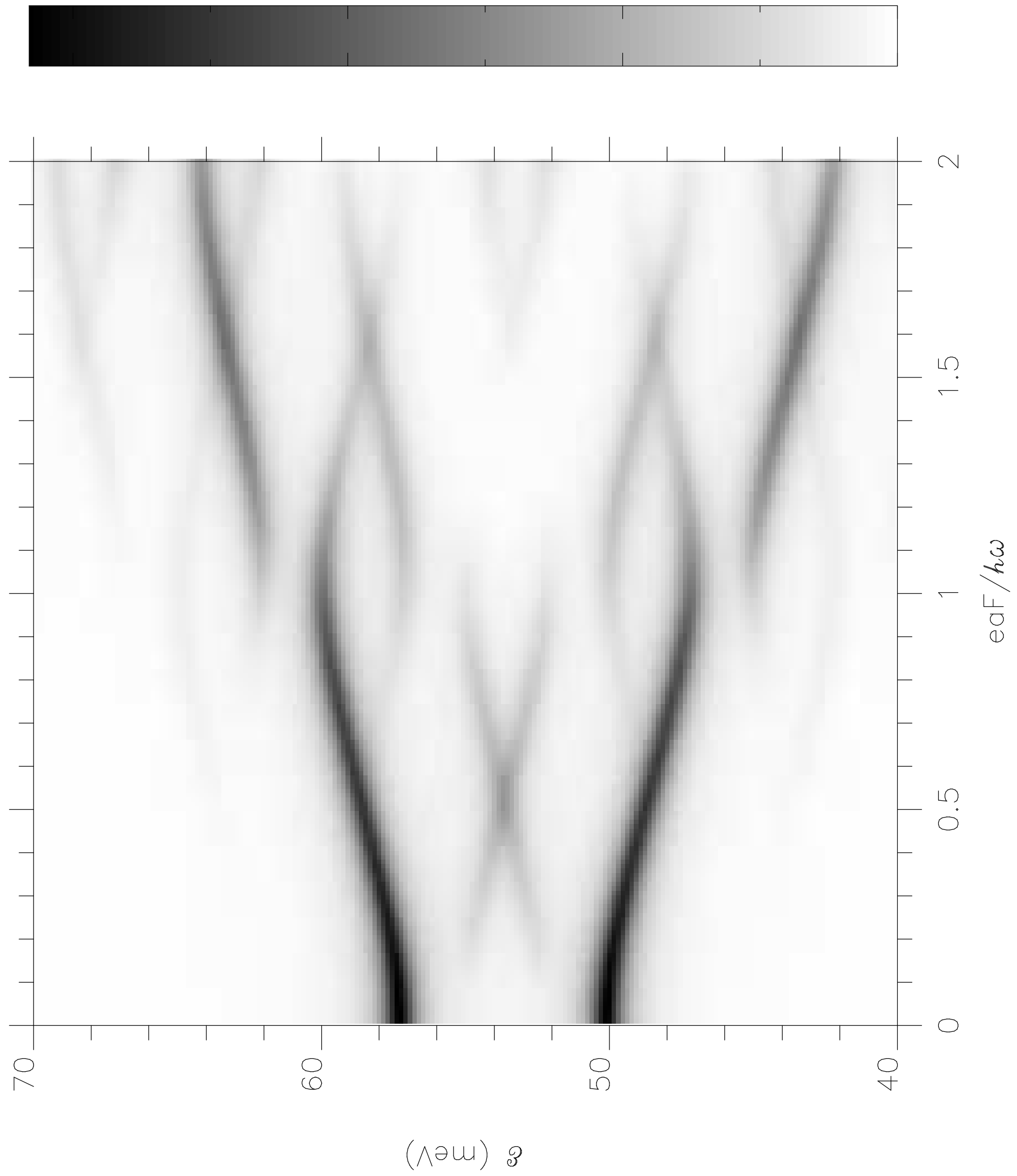


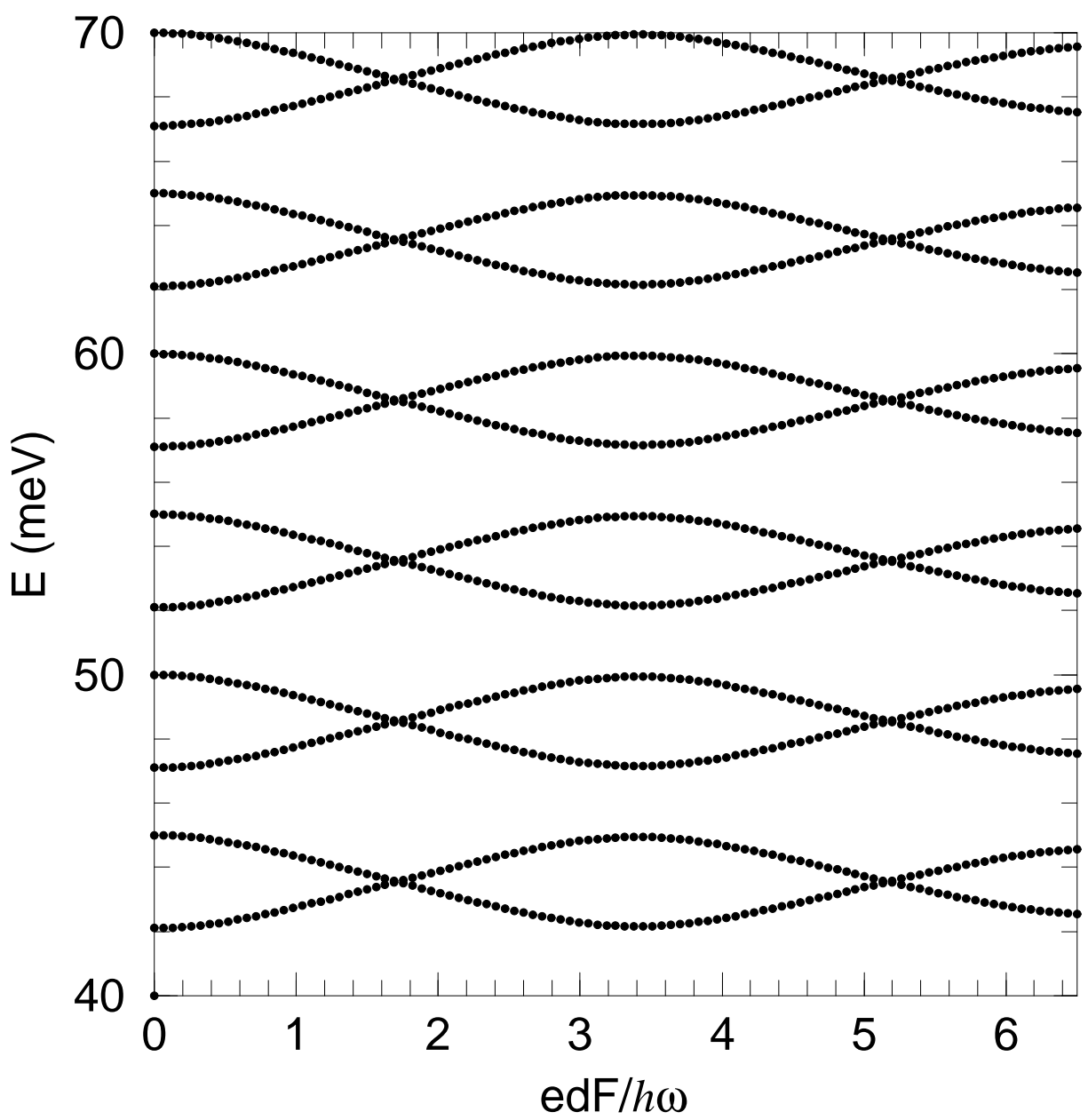

